\title{
Verification Method for Exponential Distribution and Uniform Distribution Models
}

\author{
Tang Xuemei \\ Science and Technology on Complex Systems \\ Simulation Laboratory \\ Beijing, China \\ e-mail: xuemeitang@136.com
}

\author{
Gan Bin \\ Science and Technology on Complex Systems \\ Simulation Laboratory \\ Beijing, China \\ e-mail: colebin@aliyun.com
}

\begin{abstract}
Verification method of simulation models is explored using actual output and simulation output. Verification method of static data in small-sample circumstances is focused on in this paper, and the verification method of dynamic data in small-sample circumstances is seen in literature [1]. From the probability statistical point of view, verification method of simulation models of Exponential Distribution and Uniform Distribution in small-sample circumstances is presented in this paper with regard to static data.
\end{abstract}

Keywords- Verification Method;Simulation Model; Uniform Distribution;Exponential Distribution

\section{INTRODUCTION}

Weapon system simulation is a methodology that examines and studies the real system (or conceptual system) using the model of weapon system, which can support the lifecycle of weapon system development. The fundamental goal of weapon system simulation is to analyze and research the substitution or partial substitution of the real system by the results of the simulation. Nevertheless, whether the simulation model represents a real system, and the result of the simulation system represents the function of the real system, the degree of credibility of the simulation is in question. In recent years, the verification, validation and accreditation (VV\&A) of simulation model has been significantly recognized in order to reduce the risk resulting from the errors of the simulation results of the system models and the mistakes of analysis and decision-making. The VV\&A is mentioned to spread through the whole simulation process. Actually, VV\&A is not only the guarantee for the high reliability of system simulation models, but also the fundament and precondition of the analysis and research on the real system using the simulation results. To evaluate the capacity of the real weapon system with the result of simulation experiment (as well as other experiment) require a higher level of the reliability of the weapon simulation model and therefore a higher level of the VV\&A management and techniques of the lifecycle of system development.

Verification method of simulation models will be discussed in this paper. The verification method comes after the establishment of the simulation model, and under the condition of the systematic input, compare the systematic real output and the result of simulation output, contrast the degree of the difference, and then verify the congruity of the simulated system and the real system. The outputs of the simulated system are consisted of static data and dynamic data, for example, the point error and the miss distance of the missile are all static, and the characteristic parameters such as position, velocity, azimuth, system gain, phase angle and other parameters of the missile control system are all dynamic. We need different verification methods to deal with these different data. The verification method of static data in small-sample circumstances is focused on in this paper, and the verification method of dynamic data in smallsample circumstances is seen in literature [1].

When the results of the simulation is static data, to verify whether it matches with the experimental results of the actual system, from the perspective of mathematical statistics, is to test if the results of the two experiments belong to the same probability distribution. Firstly, assume that $t_{11}, \cdots, t_{1 r_{1}}$, $t_{21}, \cdots, t_{2 r_{2}}$ obey the distribution function $F(x), G(x)$ respectively where $t_{11}, \cdots, t_{1 r_{1}}$ is the experimental data of the actual system; $t_{21}, \cdots, t_{2 r_{2}}$ is the experimental data of simulation, and $F(x), G(x)$ is unbeknown but continuous. Now, we will verify the congruity of the experimental data resulting from the simulation and the real system, which is to verify the following composite hypothesis ${ }^{[2,3,4]}$ :

$$
\mathrm{H}_{0}: F(x)=G(x) \quad \mathrm{H}_{1}: F(x) \neq G(x)
$$

We can resolve the problem about the hypothesis testing above by using non-parametric tests, such as run test, Smirnov test, ranks test and Mood test[1]. However, these test methods require a mass of experimental data, so the verification method of simulation model in small-sample circumstance will be proposed.

The experiment is influenced by the stochastic factors, so the experimental results are the appearance of stochastic variants. Determine the distribution functions, $F(x)$ and $G(x)$ regarding the stochastic variants of $t_{11}, \cdots, t_{1 r_{1}}$ and $t_{21}, \cdots, t_{2 r_{2}}$ respectively, and hypothesize in probe that the form of distribution function $F(x)$ is acknowledged, for example, $F(x)$ is normal distribution, lognormal distribution, exponential distribution, Weibull distribution or Binomial distribution. Then to testify whether $t_{11}, \cdots t_{1 r_{1}}$ obey 
the distribution function $F(x)$, the following statistic hypothesis can be drawn:

$\mathrm{H}_{0}: F(x)$ is the distribution function of the population $X$

If the form of the distribution function is acknowledged, but the parameters of the distribution are unknown, we need estimate the parameters of the distribution using $t_{11}, \cdots t_{1 r_{1}}$, and then verify the null hypothesis $\mathrm{H}_{0}, \boldsymbol{t}_{\mathbf{1 1}}, \cdots \boldsymbol{t}_{1 \boldsymbol{1}_{1}}$ obey this distribution when the null hypothesis is met, otherwise, $\boldsymbol{t}_{11}, \cdots \boldsymbol{t}_{1 r_{1}}$ don't obey this distribution. The experimental data $\boldsymbol{t}_{\mathbf{1 1}}, \cdots \boldsymbol{t}_{1 \mathbf{1}_{\mathbf{1}}}$ is modeled using Bootstrap method and Johnson method in literature [1].

The hypothesis that the form of $F(x)$ and $G(x)$ is acknowledged and the parameter of the distribution is not unknown is put forward in this paper. We can verify the validity of the simulation models by verifying the formula (1). The Exponential Distribution and Uniform Distribution that $F(x), G(x)$ obeying are discussed in this paper, and the Normal distribution or other distribution are seen in literature [2].

\section{VALIDITY TEST OF EXPONENTIAL DISTRIBUTION}

\section{DATA}

Assuming $t_{11}, \cdots, t_{1 r_{1}}, t_{21}, \cdots, t_{2 r_{2}}$ obey the distribution function $F(x), G(x)$ respectively where $t_{11}, \cdots, t_{1 r_{1}}$ is the experimental data of the actual system; $t_{21}, \cdots, t_{2 r_{2}}$ is the experimental data of simulation, and $F(x), G(x)$ is unbeknown but continuous. The next work is to verify whether $t_{11}, \cdots, t_{1 r_{1}}$ and $t_{21}, \cdots, t_{2 r_{2}}$ obey Exponential Distribution, namely, to verify whether $F(x)$ and $G(x)$ are Exponential Distribution. The statistic test of validity is followed $^{[5]}$ :

Null hypothesis $\mathrm{H}_{0}: \lambda$ is equal to constant, where $\lambda$ is distribution parameter;

Alternative hypothesis $H_{1}: \lambda$ is not equal to constant. The steps of validation are given:

\section{A. Calculate statistic} follow:

The formula of statistic of $\chi^{2}$ distribution is denoted as

$$
\begin{aligned}
& \chi_{i}^{2}=2 \sum_{k=1}^{r_{i}} \ln \frac{T_{i}}{T_{i k}} \\
& \text { where } T_{i k}=t_{i k}, k=1,2, \cdots r_{i} .
\end{aligned}
$$

We can prove that the statistic $\chi_{i}^{2}$ obey $\chi^{2}$ distribution with its degrees of freedom $\gamma=2 r_{i}$.

\section{B. Look up the quantile table of $\chi^{2}$ distribution}

With the given risk $\alpha=0.10$, and using two-tailed test, we can get the quantile of $\chi^{2}$ distribution with its degrees of freedom $\gamma_{i}$ equal to $2 r$ by looking up the table.

\section{Compare and distinguish}

$$
\text { If } \chi_{0.05}^{2}\left(2 r_{i}\right) \leq \chi_{i}^{2} \leq \chi_{0.95}^{2}\left(2 r_{i}\right) \text {, the null hypothesis } \mathrm{H}_{0}
$$

can be accepted; otherwise, the null hypothesis $\mathrm{H}_{0}$ is rejected.

\section{VERIFICATION OF EXPONENTIAL DISTRIBUTION MODEL IN SMALL-SAMPLES}

If the experimental data of the actual system and the simulation are both verified by exponential distribution test, and $t_{11}, \cdots, t_{1 r_{1}}$ obey the exponential distribution with its failure rate equal to $\lambda$, the simulation model can be verified by testing whether $t_{11}, \cdots, t_{1 r_{1}}$ and $t_{21}, \cdots, t_{2 r_{2}}$ both obey the identical exponential distribution.

Some common verification methods are introduced in literature [1], however, quantities of experimental data of the actual system are required. The verification method of the exponential distribution model in small-sample circumstances is presented in this section:

We put the samples data $t_{11}, \cdots, t_{1 r_{1}}$ and $t_{21}, \cdots, t_{2 r_{2}}$ in sequence, the result is a sequential subsamples: $t_{1} \leq t_{2} \leq \cdots \leq t_{r}$, where $\boldsymbol{r}=\boldsymbol{r}_{\mathbf{1}}+\boldsymbol{r}_{\mathbf{2}}$.

Suppose that the data $t_{11}, \cdots, t_{1 r_{1}}$ and $t_{21}, \cdots, t_{2 r_{2}}$ obey the exponential distribution with its failure rate equal to $\lambda$. Let $\partial \ell_{i}=\frac{t_{i}}{t_{r}}$, where $i=1,2, \cdots r-1$, the joint distribution density of $\partial \ell_{1}, \partial \ell_{2}, \cdots, \ell_{r-1}$ is given by

$$
f\left(\partial_{1}, \partial_{2}, \cdots, \partial_{r-1}\right)=\frac{r !}{\left(1+d_{1}+d_{2}+\cdots+d_{r-1}\right)^{r}}
$$

Proof: If $n=2$, then $\partial \ell=\frac{t_{1}}{t_{2}}$. As mentioned above, $\boldsymbol{t}_{\boldsymbol{i}}$ obeys the exponential distribution, which is given by $\boldsymbol{F}(\boldsymbol{t})=1-\exp (-\lambda \boldsymbol{t})$. So that $\forall t_{i}, \exists \alpha_{i} \in u[0,1]$ which meet

$$
t_{i}=-\lambda^{-1} \ln \left(1-\alpha_{i}\right)
$$




$$
\text { where } \alpha_{1} \leq \alpha_{2} \text {. }
$$

Let

$$
\begin{aligned}
& y_{1}=\ln \left(1-\alpha_{1}\right) \quad, \quad y_{2}=\frac{\ln \left(1-\alpha_{1}\right)}{\ln \left(1-\alpha_{2}\right)} \\
& \partial \ell=\frac{t_{1}}{t_{2}}=\frac{\ln \left(1-\alpha_{1}\right)}{\ln \left(1-\alpha_{2}\right)}, \\
& \text { we can deduce the following results }
\end{aligned}
$$

$$
\alpha_{1}=1-e^{y_{1}}, \alpha_{2}=1-e^{y_{1} / y_{2}}
$$

Note that

$$
\frac{\partial\left(\alpha_{1}, \alpha_{2}\right)}{\partial\left(y_{1}, y_{2}\right)}=\left|\begin{array}{cc}
-e^{y_{1}} & 0 \\
-\frac{1}{y_{2}} e^{\frac{y_{1}}{y_{2}}} & \frac{y_{1}}{y_{2}} e^{\frac{y_{1}}{y_{2}}}
\end{array}\right|=-\frac{y_{1}}{y_{2}} e^{y_{1}\left(1+\frac{1}{y_{2}}\right)}
$$

the density function of $\boldsymbol{y}_{1}, \boldsymbol{y}_{\mathbf{2}}$ is given as follow:

$$
f\left(y_{1}, y_{2}\right)=f\left(\alpha_{1}, \alpha_{2}\right)\left|\frac{\partial\left(\alpha_{1}, \alpha_{2}\right)}{\partial\left(y_{1}, y_{2}\right)}\right|=\frac{2 y_{1}}{y_{2}} e^{y_{1}\left(1+\frac{1}{y_{2}}\right)}
$$

We can easily get the following integral:

$$
f\left(y_{2}\right)=\int f\left(y_{1}, y_{2}\right) d y_{1}=\frac{2}{\left(1+y_{2}\right)^{2}}
$$

And as we know that $\partial \ell=y_{2}$, so

$$
f(\partial)=2-\frac{2}{1+\partial \ell}
$$

Proving as the above steps that if $n=3$, the joint distribution density function is given by:

$$
f\left(\chi_{1}, \partial \ell_{2}\right)=\frac{12}{\left(1+\partial l_{1}+\partial l_{2}\right)^{3}}
$$

Generally, if $n=r$, the joint distribution density of $\partial \ell_{1}, \partial \ell_{2}, \cdots, \partial \ell_{r-1}$ is given as formula (4). The appearance probability of the experimental data $t_{11}, \cdots, t_{1 r_{1}}$ and $t_{21}, \cdots, t_{2 r_{2}}$ can be calculated as follow:

$$
S_{P}=\int_{0}^{a_{1}} \cdots \int_{0}^{\partial_{r-1}} \frac{r !}{\left(1+d_{1}+d_{2}+\cdot \cdot+d_{r-1}\right)^{r}} \mathrm{~d} d_{1} \mathrm{~d} d_{2} \cdot \cdot \mathrm{d} d_{r-1}
$$

We can calculate the critical point $S_{k p}$ corresponding to different failure $r$ of data and different confidence level using computer and Monte-Carlo method.

If $S_{p} \geq S_{k p}$, then the hypothesis that data $t_{11}, \cdots, t_{1 r_{1} \text { and }} t_{21} \cdots, t_{2 r_{2}}$ obey the exponential distribution with failure rate equal to $\lambda$ is not true under the given confidence level, that is, the simulation model is unauthentic. Otherwise, the simulation model is authentic.
IV. VERIFICATION OF UNIFORM DistRIBUTION MODEL IN SMALL-SAMPLES

If the experimental data $t_{11}, t_{12}, \cdots, t_{1 r_{1}}$ of the actual system are the uniform distribution $u[a, b]$ variables, and $t_{21}, t_{22}, \cdots, t_{2 r_{2}}$ are the experimental data of simulation, to verify the simulation model is to testify the consistency of $\boldsymbol{t}_{11}, \boldsymbol{t}_{12}, \cdots, \boldsymbol{t}_{1 r_{1} \text { and }} t_{21}, t_{22}, \cdots, t_{2 r_{2}}$.

The verification method of the consistency of the uniform distribution data in small-sample circumstances is discussed in this section. The test steps are presented as follow ${ }^{[7,8]}$ :

Put the data $t_{11}, \cdots, t_{1 r_{1}}$ and $t_{21}, \cdots, t_{2 r_{2}}$ in sequence and the result is sequential sub-samples: $t_{1} \leq t_{2} \leq \cdots \leq t_{r}$, where $r=r_{1}+r_{2}$

Suppose that the experimental data $t_{11}, \cdots, t_{1 r_{1}}$ and $t_{21}, \cdots, t_{2 r_{2}}$ come from the uniform distribution $u[a, b]$. Let

$$
\partial \ell_{i}=\frac{\boldsymbol{t}_{\boldsymbol{i}}-\boldsymbol{t}_{\mathbf{1}}}{\boldsymbol{t}_{\boldsymbol{r}}-\boldsymbol{t}_{\mathbf{1}}} i=1,2, \cdots r-1
$$

the joint distribution density of $\partial \ell_{1}, \partial \ell_{2}, \cdots, \partial \ell_{r-1}$ is given by

$$
f\left(\partial \ell_{1}, \partial \ell_{2}, \cdots, \partial \ell_{r-1}\right)=1
$$

Proof: As we know, $\boldsymbol{t}_{\boldsymbol{i}}$ are the variables of the uniform distribution $u[a, b]$, so $\forall t_{i}, \exists \alpha_{i} \in u[0,1]$ which meet

$$
t_{i}=a+(b-a) \alpha_{i}
$$

where $\alpha_{1} \leq \alpha_{2} \leq \ldots \leq \alpha_{r}$. And the statistic $\partial \ell_{i}$ can be deduced as follow:

$$
\partial \ell_{i}=\frac{t_{i}-t_{1}}{t_{r}-t_{1}}=\frac{\alpha_{i}-\alpha_{1}}{\alpha_{r}-\alpha_{1}}, i=1,2, \cdots r-1
$$

Because the statistic ${ }^{\partial l_{i}}$ are independent of the interval parameters $a, b$, and dependent of the random variables $\alpha_{i}(i=1,2, \cdots, r-1)$ in $u[0,1]$, using the method discussed in section 3 , the joint distribution density of $d_{1}, d_{2}, \cdots, \ell_{r-1}$ can be denoted by (13).

The appearance probability of the experimental data $t_{21}, t_{22}, \cdots, t_{2 r_{2}}$ is calculated ${ }^{[9]}$ :

$$
S_{P}=\int_{0}^{\partial_{1}} \cdots \int_{0}^{\partial_{r-1}} \mathbf{d} \partial_{1} \mathbf{d} \partial l_{2} \cdots \mathbf{d} \partial_{r-1}=\partial_{1} \partial_{2} \cdots d_{r-1}
$$

We can calculate the critical point $S_{k p}$ corresponding to different amount $r$ of measuring data and different 
confidence level using computer to simulate $u[0,1]$. The results are seen Table I.

TABLE I. TABLE TyPE STyles

\begin{tabular}{|c|c|c|c|c|}
\hline \multirow{2}{*}{$\alpha$} & \multicolumn{4}{|c|}{$S_{K P}$} \\
\cline { 2 - 5 } & $N=3$ & $N=4$ & $N=5$ & $N=6$ \\
\hline 0.050 & 0.050 & 0.01173 & 0.00157 & 0.00043 \\
\hline 0.100 & 0.100 & 0.02140 & 0.00441 & 0.00123 \\
\hline 0.150 & 0.150 & 0.03575 & 0.00762 & 0.00226 \\
\hline 0.200 & 0.200 & 0.05077 & 0.01072 & 0.00379 \\
\hline 0.250 & 0.250 & 0.06262 & 0.01425 & 0.00582 \\
\hline 0.300 & 0.300 & 0.08212 & 0.02173 & 0.00832 \\
\hline
\end{tabular}

Finally, we can get the critical point $S_{k p}$ under the given $\alpha$ and $r$. If $S_{p} \geq s_{k p}$, then the hypothesis that data $t_{11}, \cdots, t_{1 r_{1}}$ and $t_{21}, \cdots, t_{2 r_{2}}$ obey the uniform distribution $u[a, b]$ is not true under the given confidence level, that is, the simulation model is unauthentic. Otherwise, the simulation model is authentic.

\section{CONCLUSIONS}

Accounting for the characteristics of the system simulation such as credibility, security and economical efficiency, the verification methods of the simulation model are discussed in this paper using the actual output of the real system and the results of the simulation. Our methods are appropriate for the verification of the static data in smallsample circumstances.

\section{ACKNOWLEDGMENT}

The authors of this paper would like to thank Professor Zhang Jinhuai, Executive Director for Operational Research Societies., who is long engaged in researching and teaching in aerocraft test statistics. The authors of this paper would also like to thank both $\mathrm{Hu}$ Zhengdong, $\mathrm{PhD}$, and Wang Liangchun, $\mathrm{PhD}$ for their encouraging support of the testing.

\section{REFERENCES}

[1] Tang Xue-mei. "Test Analysis and Evaluation of Weapon Systems in Small-Sample Circumstances". National Defense Industry Publishing Company, Beijing, 2001.

[2] Tang Xue-mei. "Consistency Test Analysis of Simulated Data in Small-Sample Circumstances". System Engineer and Electronical Technology, Volume 102001

[3] Duan Xiao-jun, Wang Zheng-ming. "Applicability of Bootstrap Method in Small Sample Case". Journal of Ballistics, 2003,15(3).

[4] Li Bao-Sheng, He Hong-qing. "Credibility bounds study of mission time reliability in Weibull distribution". Journal of Propulsion Technology,2001,22(5).

[5] Shang Chang-an, Liu Xing-tang, Wu Hao. "Main Traits and Confidence Assessment Measures of Military Large-scale and Complicated Simulation System". Journal of System Simulation.2002,14(5).
[6] Hu Wei-wen, Yuan Bing-cheng, Yang Peng. "Research on Decisionmaking Analysis about Simulation Model Credibility". Journal of System Simulation. 2008, 20(12).

[7] Feng Yun-wen, Huang Wei. "The Semiempirical Evaluation Method for Extreme Small Sample Test”, ACTA AERONAUTICA ET ASTRONAUTICA SINICA,2004,25(5).

[8] Li Hong-shuang, Lv Zhen-zhou. "Bootstrap Method for Confidence Lower Limit Estimation of Population Percentile and Reliability in Small Number of Test-Sample". ACTA AERONAUTICA ET ASTRONAUTICA SINICA,2006,27(5).

[9] Li Yun-feng. "Verification,validation and accreditation on digital simulation model". Journal Of Central South University ( Science and Technology) ,2004,35(2)

[10] Wang Rui-ping, Zhang Wu-zhou. "Controlled Trajectory Modelling Simulation Debugging and Verification of Surface to Air Missile". Fire Control \& Command Control.2010,35(6). 